\title{
Robust Control of Reservoir Release Discharge Using Precipitation Data
}

\author{
Koichi Unami ${ }^{1}$, Shigeki Nakanishi ${ }^{2}$ and Toshihiko Kawachi ${ }^{3}$
}

\begin{abstract}
A linear control system is developed and is applied to automatic control of reservoir release discharge using precipitation data. A nominal generator, which is an estimated runoff model from precipitation to inflow into the reservoir, is assumed to be obtained. The control system has three controllers to cancel out external disturbances, to handle wind-up phenomena in the release discharge, and to stabilize the entire system under observation errors. Demonstrative calculations are executed using flood data observed at an existing dam. The results show that three controllers mends the variation in the reservoir storage complementing each other.
\end{abstract}

\section{Introduction}

Automatic regulation of release discharge from a reservoir is a key subject to achieving its flexible and efficient operations. In order to keep the storage at a targeted value, the release discharge should be determined according to a well established rule. The linear control theory has been applied to a water storage model which describes reservoirs (Nakanishi et al., 1997) as well as open channel networks (Unami and Kawachi, 1997).

Here, a novel control system is developed for a reservoir where precipitation data is available in terms of real time operation. The control system essentially differs from the earlier works in the following two respects. In the first sense, the control errors are taken into account to prevent wind-up phenomena, or awkward expansion of a control variable. And in the second sense, the external disturbances are related to observable references by a generator, which is also represented by a linear dynamical model and may include modeling errors. In this context, three controllers are designed; one is a feed-forward controller to cancel out the external disturbances assuming that there is no modeling error in the generator, one is a controller for anti wind-up, and the other one is a feed-back controller to stabilize the control system under observation errors and to bound variations in the storage volume of the reservoir. Thus, the system is an extension of the two-degree-of freedom system which consists of a feed-back controller and a feed-back controller (Horowitz, 1963). The robust control theory, which rigorously deals with the modeling errors as well as

\footnotetext{
${ }^{1}$ Instructor, Graduate School of Agricultural Science, Kyoto University, Kitashirakawa-oiwake-cho, Sakyo-ku, Kyoto, 606-8502 Japan

${ }^{2}$ Design Division, Agricultural Structure Improvement Bureau, Ministry of Agriculture, Forestry and Fisheries, 12-1 Kasumigaseki, Chiyoda-ku, Tokyo, 100-8950 Japan

${ }^{3}$ Professor, Graduate School of Agricultural Science, Kyoto University, Kitashirakawa-oiwake-cho, Sakyo-ku, Kyoto, 606-8502 Japan
}

the external disturbances, is applied to designing the feed-back controller. The controllers designed are tested in a reservoir using two sets of data in floods.

\section{Water storage model}

Throughout the mathematical description of the control system, the same notation is used for representing a variable both in the time and the frequency domains. The frequency is denoted by $s$.

A reservoir is modeled as the linear system given by the state equation

$$
\frac{d x}{d t}=u+q
$$

with the observation equation

$$
y=x
$$

where $t=$ time, $x=$ state variable which represents the storage volume deviation, $u=$ control variable which represents the release discharge deviation, $q$ $=$ external disturbance, and $y=$ observation output.

The external disturbance $q$, which is the external inflow discharges into the reservoir, is assumed to be generated by a generator $G$ from an observable reference $r$. When the reservoir is fed by the runoff resulting from the precipitation as illustrated in Figure 1, the generator $G$ and the reference $r$ are a runoff model and precipitation data, respectively.

\section{Errors in the model}

The modeling error in the generator $G$ and the control error are taken into account in the controller design.

The generator $G$, whose definite expression is hardly obtained, is perturbed from a nominal generator $G_{0}$, which is written as

$$
G_{0}=\frac{k}{s+a}
$$




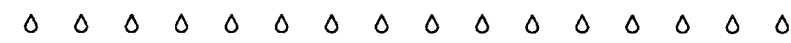

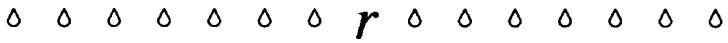

$\begin{array}{llllllllllllllll}0 & 0 & 0 & 0 & 0 & 0 & 0 & 0 & 0 & 0 & 0 & 0 & 0 & 0 & 0 & 0\end{array}$

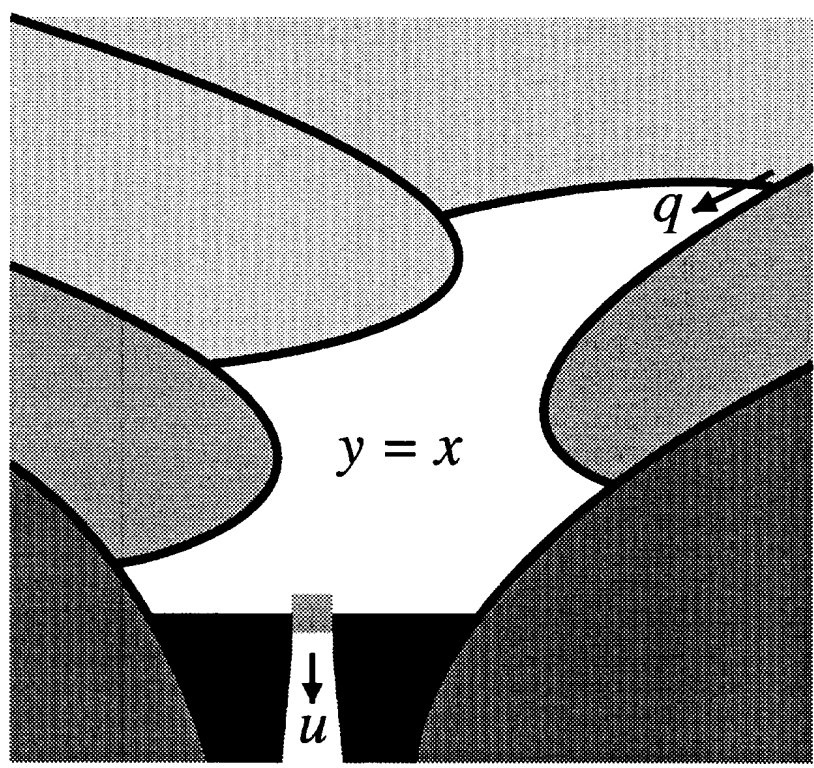

Figure 1: Sketch of reservoir

where $k=$ load coefficient, and $a=$ decay coefficient. In the true generator $G, k$ and $a$ are assumed to deviate as $k+\Delta^{k}$ and $a+\Delta^{a}$ with deviation parameters $\Delta^{k}$ and $\Delta^{a}$ whose maximum absolute values are prescribed. Then, the generator $G$ as the transfer function from $r$ to $q$ is expressed as the block diagram shown in Figure 2.

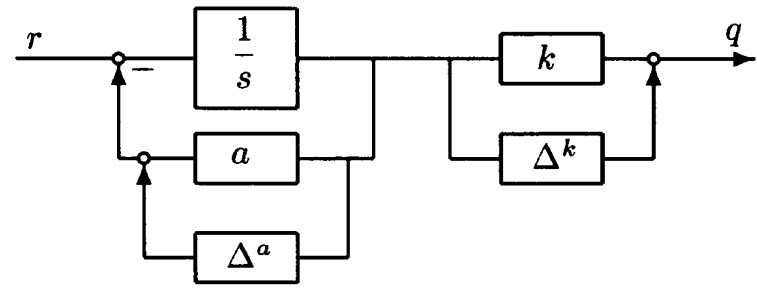

Figure 2: Block diagram of gengerator $G$

The control error is mostly due to the saturation of control variables. If a gate is fully open, the discharge cannot be controlled because of free overflow. Thus, the control variable $u$ is assumed to be multiplicatively perturbed from a nominal control variable $u_{1}$ as

$$
u=\left(1+\Delta^{u}\right) u_{1}
$$

where $\Delta^{u}=$ deviation parameter for $u$.
4 Design of controllers

The Doyle's notation (Doyle et al., 1989) is used for the system matrix. For example, $\left[\begin{array}{l|l}G_{11} & G_{12} \\ \hline G_{21} & G_{22}\end{array}\right]$ denotes the transfer matrix $G_{21}\left(s I-G_{11}\right)^{-1} G_{12}+$ $G_{22}$ for matrices $G_{i j}(i, j=1,2)$ of compatible dimensions and the unit matrix $I$.

Let the reference $r$, the actual control variable $u$, and the observation output $y$ be known. In the control system whose block diagram is shown in Figure 3 , controllers $K_{G}, K_{C}$, and $K_{R}$ are designed to determine the nominal control variable $u_{1}$ which stabilizes the entire system.

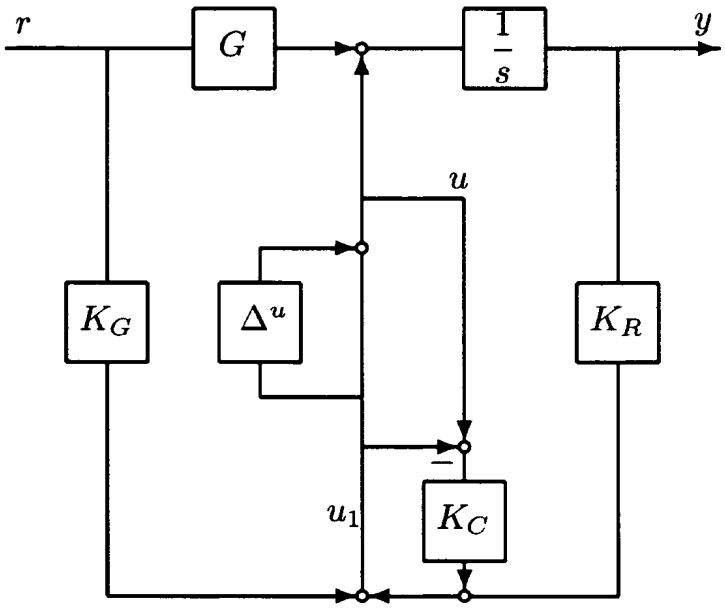

Figure 3: Block diagram of control system

4.1 Controller $K_{G}$

The controller $K_{G}$ is chosen as

$$
K_{G}=-G_{0} \stackrel{s}{=}\left[\begin{array}{l|l}
-a & 1 \\
\hline-k & 0
\end{array}\right]
$$

to cancel out the external disturbance if there is no error in the whole system.

\subsection{Controller $K_{C}$}

Extracting the control error characteristics as an $H_{\infty}$ standard problem as shown in Figure 4, the controller $K_{C}$ is determined to make the $H_{\infty}$ norm of the closed-loop transfer function matrix, which is equal to $\operatorname{diag} \Delta^{u}$, less than unity. The class of such $K_{C}$ is characterized by

$$
K_{C}=\frac{N(s)}{\Delta^{u}}
$$

where $N(s)=$ any $B H_{\infty}$ transfer function matrix. 


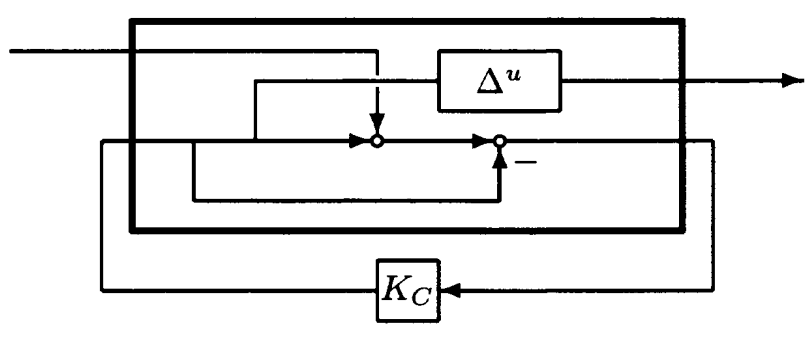

Figure 4: $H_{\infty}$ standard plant for $K_{C}$

\subsection{Controller $K_{R}$}

The algebraic design method is employed to obtain the controller $K_{R}$. An $H_{\infty}$ standard problem is formulated as expressed in Figure 5 to bound the $H_{\infty}$ norm of the closed-loop transfer function from an input disturbance vector $\left[\begin{array}{l}w_{1} \\ w_{2} \\ w_{3}\end{array}\right]$ to an error signal vector $\left[\begin{array}{l}z_{1} \\ z_{2} \\ z_{3}\end{array}\right]$ taking the reference $r$ as the input disturbance $w_{1}$ and using $w^{y}$, constant weights to scale the observation output $y$ as the error signal $z_{3}$. A state space representation of the generalized plant in the $H_{\infty}$ standard problem is obtained as

$$
\left[\begin{array}{cccc}
\frac{\Delta^{a}}{s+a} & \frac{-\Delta^{a}}{s+a} & 0 & 0 \\
\frac{\Delta^{k}}{s+k} & \frac{-\Delta^{k}}{s+k} & 0 & 0 \\
0 & \frac{-w^{y} \Delta^{k}}{s(s+a)} & \frac{w^{y}}{s} & \frac{-w^{y}}{s} \\
0 & \frac{-\Delta^{k}}{s(s+a)} & \frac{1}{s} & \frac{1}{s}
\end{array}\right] \stackrel{s}{=}\left[\begin{array}{c|cc}
A_{0} & B_{1} & B_{2} \\
\hline C_{1} & {[0]} & {[0]} \\
C_{2} & {[0]} & {[0]}
\end{array}\right]
$$

where $A_{0}=\left[\begin{array}{cccc}-a & 0 & 0 & 0 \\ 0 & -a & 0 & 0 \\ 0 & 0 & 0 & k \\ 0 & 0 & 0 & -a\end{array}\right]=$ state matrix, $B_{1}=\left[\begin{array}{ccc}1 & -1 & 0 \\ 1 & -1 & 0 \\ 0 & 0 & 1 \\ 0 & -1 & 0\end{array}\right]=$ disturbance distribution matrix, $B_{2}=\left[\begin{array}{l}0 \\ 0 \\ 1 \\ 0\end{array}\right]=$ control distribution matrix, $C_{1}=\left[\begin{array}{cccc}\Delta^{a} & 0 & 0 & 0 \\ 0 & \Delta^{k} & 0 & 0 \\ 0 & 0 & w^{y} & 0\end{array}\right]=$ error output matrix, and $C_{2}=\left[\begin{array}{llll}0 & 0 & 1 & 0\end{array}\right]=$ observation output matrix.

Since all the components are zero matrix in the 2-2 block of the system matrix in Eqn. 7, the class of all stabilizing controllers cannot be identified as in Glover and Doyle (1988). However, Sampei et

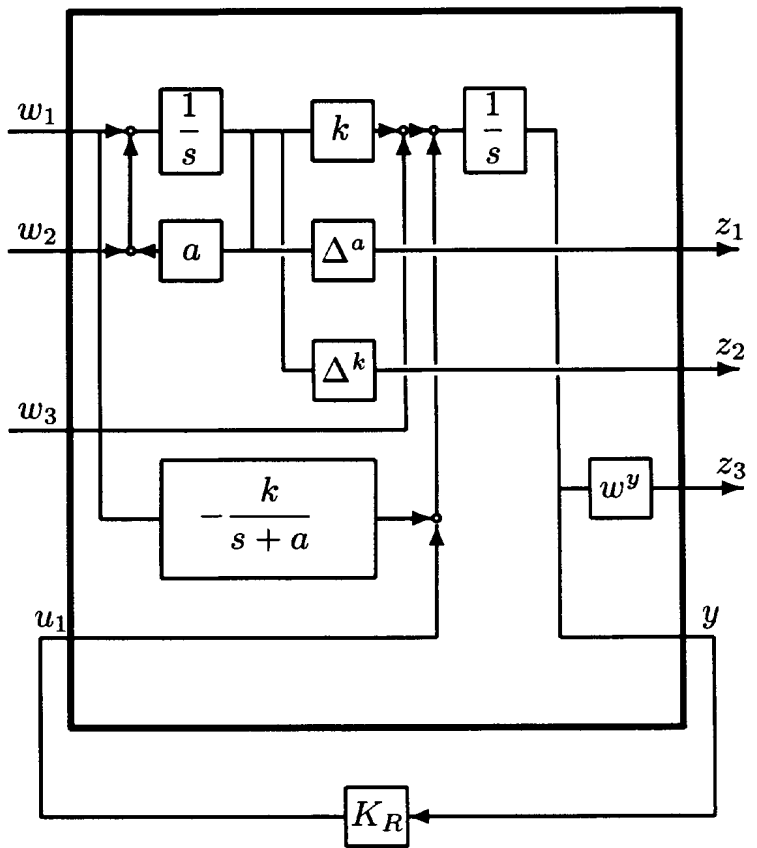

Figure 5: $H_{\infty}$ standard plant for $K_{R}$

al. (1990) deduce an existence theorem and take an algebraic approach similar to Zhou and Khargonekar (1988) as follows.

A strictly proper controller stabilizes the system and makes the $H_{\infty}$ norm of the closed-loop transfer function less than unity if and only if the following conditions are satisfied.

1. There exists $F$ such that there exists a positive definite solution $X$ to the algebraic Riccati inequality

$$
X A_{X}+A_{X}^{T} X+C_{1}^{T} C_{1}+X B_{1} B_{1}^{T} X<0
$$

where $A_{X}=A_{0}+B_{2} F$.

2. There exists $L$ such that there exists a positive definite solution $Y$ to the algebraic Riccati inequality

$$
Y A_{Y}^{T}+A_{Y} Y+B_{1} B_{1}^{T}+Y C_{1}^{T} C_{1} Y<0
$$

where $A_{Y}=A_{0}+L C_{2}$.

3. The maximum singular value of $(X Y)$ is less than unity.

Moreover, one of such strictly proper controller is given by

$$
K_{R}=\left[\begin{array}{c|c}
\hat{A} & -Z Y^{-1} L \\
\hline F & 0
\end{array}\right]
$$


where $Z=\left(Y_{\infty}^{-1}-X_{\infty}\right)^{-1}$, and

$$
\hat{A}=A_{X}+B_{1} B_{1}^{T} X-Z\left(E+F^{T} B_{2}^{T} X-Y^{-1} L C_{2}\right)
$$

with $E=-\left(X A_{X}+A_{X}^{T} X+C_{1}^{T} C_{1}+X B_{1} B_{1}^{T} X\right)$. Numerical solutions of the Riccati equations

$$
X A_{X}+A_{X}^{T} X+C_{1}^{T} C_{1}+X B_{1} B_{1}^{T} X+\varepsilon I=0
$$

and

$$
Y A_{Y}+A_{Y}^{T} Y+B_{1} B_{1}^{T}+Y C_{1}^{T} C_{1} Y+\varepsilon I=0
$$

determine $X$ and $Y$, respectively, in practical design.

\section{Application to reservoir operation in flood} The controllers are applied to determining the release discharge of a reservoir when a flood wave enters into it. Two sets of data D-1 and D-2 observed at an existing dam whose catchment area is of 32.45 $\mathrm{km}^{2}$ are used for identifying the runoff model. Identification procedures using the least square method yield the runoff models $\frac{0.2012}{s+6.899}$ and $\frac{0.0885}{s+3.506}$ for D-1 and D-2, respectively. Then, the nominal generator $G_{0}$ is taken as $\frac{0.15}{s+5}$ with the deviation parameters $\Delta^{k}=0.10$ and $\Delta^{a}=3.4$. The Bode plots of the runoff models and the nominal generator $G_{0}$ are depicted in Figure 6. Setting $\varepsilon=10^{-2}$, $F=\left[\begin{array}{llll}0 & 0 & -5 & 0\end{array}\right]$, and $L=\left[\begin{array}{c}0 \\ 0 \\ -1 \\ -1\end{array}\right]$, the controller $K_{R}$ is obtained from Eqn.(10).

Simulation runs are executed for 5 cases which include different combination of controllers as shown in Table. The maximum release discharge is $1 \times 10^{7}$ $\mathrm{m}^{3} /$ day and the control variable saturates when it becomes so large. Results for flood data D-1 and D2 are depicted in Figures 7 and 8, respectively, and show that the controller $K_{C}$ is necessary for settling the storage volume after the saturation of the control variable occurs. The role of the controller $K_{R}$ is to stabilize the entire system.

\section{Conclusions}

The robust control system for operation of reservoir release discharge is designed. The system considers a generator, which is the runoff model from precipitation to inflow into the reservoir. The three controllers guarantee robustness when the generator includes the modeling errors, robustness under the control errors, and bounded variations in storage volumes in the sense of the $H_{\infty}$ control problem. The specifications are verified in the simulations, which demonstrate excellent regulation performance.

The authors thank the Yasugawa land improvement association for providing the data.

\begin{tabular}{c|c}
\hline Case 1 & No controller \\
\hline Case 2 & $K_{G}$ \\
\hline Case 3 & $K_{G}$ and $K_{C}$ \\
\hline Case 4 & $K_{G}$ and $K_{R}$ \\
\hline Case 5 & $K_{G}, K_{C}$, and $K_{R}$ \\
\hline
\end{tabular}

Table 1: Combination of controllers for reservoir operation

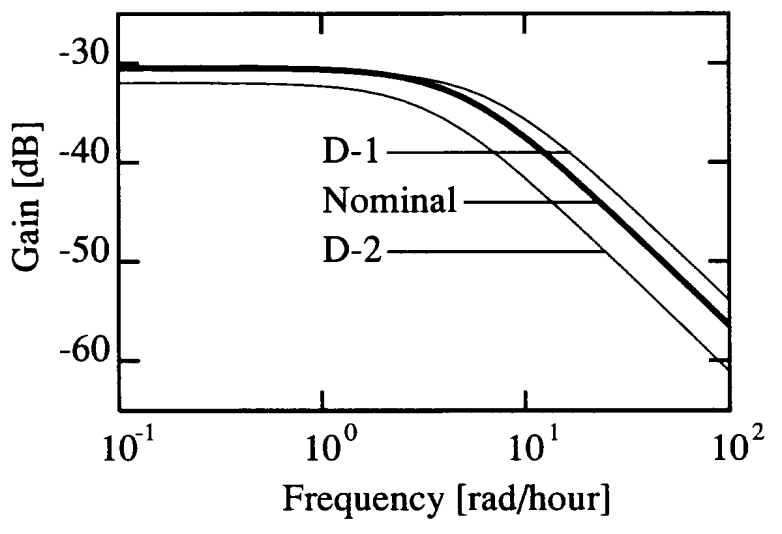

Figure 6: Bode plots

\section{References}

[1] Doyle, J.C., Glover, K., Khargonekar, P.P., and Francis, B.A. (1989): State-space solutions to standard $H_{2}$ and $H_{\infty}$ control problems, Transactions on A utomatic Control, IEEE, 34(8), pp.831-847.

[2] Glover, K., and Doyle, J.C. (1988): State-space formulae for all stabilizing controllers that satisfy an $H_{\infty}$-norm bound and relations to risk sensitivity, Systems \& Control Letters, 11, pp.167-172.

[3] Horowitz, I.M. (1963): Synthesis of Feedback Systems, Academic Press, pp.246-298.

[4] Nakanishi, S., Unami, K., and Kawachi, T. (1997): Automatic reservoir management using precipitation data, Proc. of 5th Japan International Rainwater Catchment Systems Association, pp.11-16. (in Japanese).

[5] Sampei, M., Mita, T., and Nakamichi, M. (1990): An algebraic approach to $H_{\infty}$ output feedback control problems, Systems \& Control Letters, 14, pp.13-24.

[6] Unami, K., and Kawachi, T. (1997) Rainwater Conveyance in Channel Systems, Proc. of 5th Japan International Rainwater Catchment Systems Association, pp.91-92.

[7] Zhou, K., and Khargonekar, P.P. (1988): An algebraic Riccati equation approach to $H_{\infty}$ optimization, Systems \& Control Letters, 11, pp.85-91. 

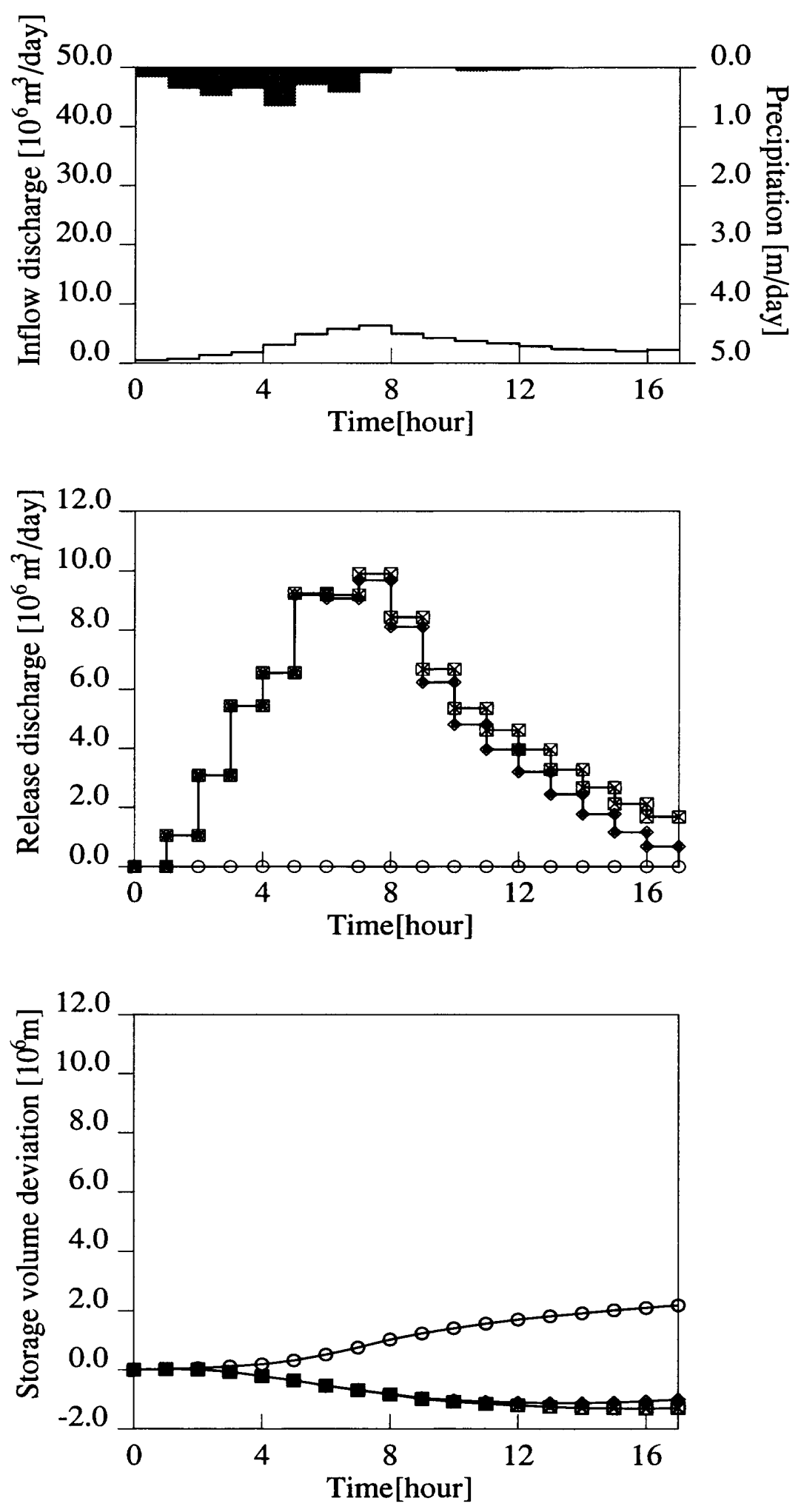

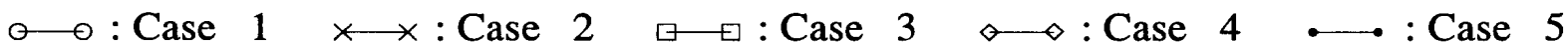

Figure 7: Simulation results for flood data D-1 

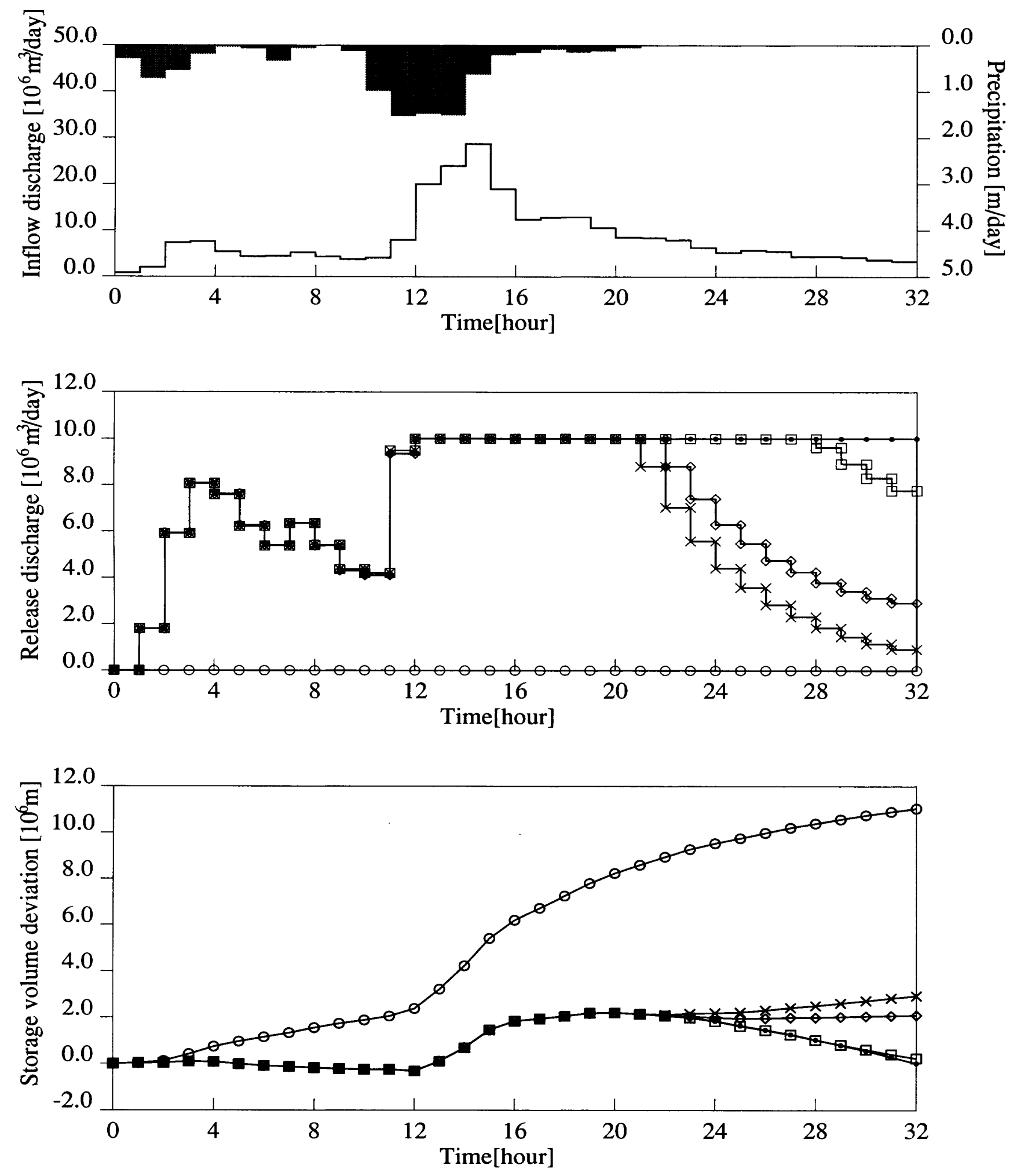

$\diamond$ :Case $1 \times \times$ : Case $2 \boxminus \bullet:$ Case $3 \diamond$ :Case $4 \bullet$ : Case 5

Figure 8: Simulation results for flood data D-2 